\title{
PRODUCT FORMULAS AND CONVOLUTIONS FOR ANGULAR AND RADIAL SPHEROIDAL WAVE FUNCTIONS
}

\author{
WILLIAM C. CONNETT, CLEMENS MARKETT, AND ALAN L. SCHWARTZ
}

\begin{abstract}
Product formulas for angular spheroidal wave functions on $[0, \pi]$ and for radial spheroidal wave functions on $[0, \infty)$ are presented, which generalize results for the ultraspherical polynomials and functions as well as for the Mathieu functions. Although these functions cannot be given in closed form, the kernels of the product formulas are represented in an explicit, and surprisingly simple way in terms of Bessel functions so that the exact range of positivity can easily be read off. The formulas are used to introduce two families of convolution structures on $[0, \pi]$ and $[0, \infty)$, many of which provide new hypergroups. We proceed from the fact that the spheroidal wave functions are eigenfunctions of Sturm-Liouville equations of confluent Heun type and employ a partial differential equation technique based on Riemann's integration method.
\end{abstract}

\section{INTRODUCTION}

One of the richest families of special functions arise in a natural way when the three dimensional Helmholtz equation $\left(\left(\Delta^{2}+k^{2}\right) W=0\right.$, also called the reduced wave equation) is solved by separation of variables in a coordinate system with concentric families of spheroids and hyperboloids as level surfaces [E, Chapter 16, AS, Chapter 21, Mi, 3.1, MSp] (the last reference contains some excellent figures). If $W(x, \theta, \phi)=U(x) V(\theta) \exp ( \pm \operatorname{im} \phi)$, then the Helmholtz equation separates into one of the following systems of two ordinary differential equations:

$$
\begin{gathered}
U^{\prime \prime}+(\operatorname{ctnh} x) U^{\prime}-\left[h-(k c \sinh x)^{2}+(m \operatorname{csch} x)^{2}\right] U=0 \\
(0<x<\infty), \\
V^{\prime \prime}+(\operatorname{ctn} \theta) V^{\prime}+\left[h+(k c \sin \theta)^{2}-(m \csc \theta)^{2}\right] V=0 \\
(0<\theta<\pi),
\end{gathered}
$$

Received by the editors February 22, 1991 and, in revised form, May 1, 1991.

1980 Mathematics Subject Classification (1985 Revision). Primary 33E10; Secondary 34B25, 43A10, 43A62. lation.

Key words and phrases. Spheroidal wave functions, convolution, hypergroup, generalized trans-

The work of the first and third author reported here was partially supported by the National Science Foundation (Grant no. DMS-9005999).

This paper was initiated while the second-named author was visiting the Department of Mathematics and Computer Science at the University of Missouri-St. Louis. He wishes to express his gratitude to that department for its hospitality. 


$$
\begin{gathered}
U^{\prime \prime}+(\tanh x) U^{\prime}-\left[h-(k c \cosh x)^{2}-(m \operatorname{sech} x)^{2}\right] U=0 \\
(0<x<\infty), \\
V^{\prime \prime}+(\operatorname{ctn} \theta) V^{\prime}+\left[h-(k c \sin \theta)^{2}-(m \csc \theta)^{2}\right] V=0 \\
(0<\theta<\pi),
\end{gathered}
$$

where $c$ is a parameter of the coordinate system and $h$ and $m$ are separation constants. The first system arises from using the prolate spheroidal coordinates, the second from using the oblate spheroidal coordinates. Solutions to the first equation in each pair are referred to as radial solutions; solutions to the second equation in each pair are called angular (or periodic) solutions. The two equations inside each pair are related to each other by the imaginary change of variables, $x \rightarrow i \theta, x \rightarrow i(\theta-\pi / 2)$, respectively.

By a transformation of the dependent variable $V(\theta)=\sin ^{m} \theta S(\theta)$ the two equations (1.1b) and (1.2b) can be written in the normalized form

$$
S^{\prime \prime}+\frac{2 \alpha+1}{\tan \theta} S^{\prime}+\left[\lambda-\gamma \sin ^{2} \theta\right] S=0 \quad(0<\theta<\pi)
$$

where $\alpha=m$ and $\gamma=\mp(k c)^{2} \quad(\gamma<0$ for prolate, $\gamma>0$ for oblate). With the addition of the boundary conditions $S^{\prime}(0)=S^{\prime}(\pi)=0$ and the normalizing condition $S(0)=1$ this becomes a Sturm-Liouville problem with a discrete spectrum of eigenvalues. The corresponding eigenfunctions are called the angular spheroidal wave functions $\left\{S_{n}^{\alpha, \gamma}(\theta)\right\}_{n \in \mathbb{N}_{0}}\left(\mathbb{N}_{0}=\{0,1,2, \ldots\}\right)$ and are dealt with in this paper for all $\alpha \geq-1 / 2$ and $\gamma \in \mathbb{R}$, each choice of $(\alpha, \gamma)$ leading to a different family of orthogonal functions. Moreover, we will consider the radial prolate spheroidal wave functions $\left\{\mathscr{S}_{\lambda}^{\alpha, \gamma}(x)\right\}_{\lambda \geq 0}$ for $\alpha \geq-1 / 2$, $\gamma \leq 0$, which are eigenfunctions of the Sturm-Liouville equation

$$
\mathscr{S}^{\prime \prime}+\frac{2 \alpha+1}{\tanh x} \mathscr{S}^{\prime}+\left[\lambda^{2}+(\alpha+1 / 2)^{2}-\gamma \sinh ^{2} x\right] \mathscr{S}=0 \quad(0<x<\infty)
$$

satisfying the initial conditions $\mathscr{S}(0)=1, \mathscr{S}^{\prime}(0)=0$. Equation (1.4) is obtained from (1.1a) by the transformation $U(x)=\sinh ^{m} x \mathscr{S}(x)$ (with $m=$ $\left.\alpha,-h=\lambda^{2}+1 / 4\right)$.

There are some well-known particular cases covered by equations (1.3) and (1.4). If $\gamma=0$ then (1.3) has as its eigenvalues $\lambda=n(n+2 \alpha+1)$ with corresponding eigenfunctions $P_{n}^{(\alpha+1 / 2)}(\cos \theta) / P_{n}^{(\alpha+1 / 2)}(1)$, where $P_{n}^{(\alpha+1 / 2)}$ is the ultraspherical polynomial of order $\alpha+1 / 2$ and degree $n$. In this case, the eigenfunctions of (1.4) reduce to the ultraspherical functions on the positive half-line. When $\alpha=\nu-1 / 2$ and $\gamma \neq 0,(1.3)$ is called the Mathieu equation if $\nu=0$ and the associated Mathieu equation if $\nu \in \mathbb{N}$. The counterparts associated with (1.4) are sometimes referred to as the respective "modified" equations (cf. Mclachlain [M]).

A great deal of work has been done with spheroidal wave functions; some standard references are Abramowitz and Stegun [AS], Arscott [A1], Erdelyi [E], Flammer [F], Meixner and Schäfke [MS], and Meixner, Schäfke, and Wolf [MSW], where the extensive literature up to 1979 is cited, and Stratton, et al. [SM]. Nevertheless, one of the most useful and far-reaching identities in the study of orthogonal functions and expansions, the product formula, had not been found for the spheroidal wave functions except in the cases $\alpha=-1 / 2$ or 
$\gamma=0$. It is the main purpose of this paper to establish the product formula for the angular case in the form

$$
S_{n}^{\alpha, \gamma}(\theta) S_{n}^{\alpha, \gamma}(\phi)=\int_{0}^{\pi} S_{n}^{\alpha, \gamma}(\xi) d \sigma_{\theta, \phi}(\xi) \quad(0<\theta, \phi<\pi)
$$

where for each $(\theta, \phi), \sigma_{\theta, \phi}=\sigma_{\theta, \phi}^{\alpha, \gamma}$ is an explicitly given Borel measure which does not depend on $n \in \mathbb{N}_{0}$ (see Theorem 1 below). For later discussion we also include the result in the limiting Mathieu case $\gamma=0$ which was first stated by Meixner [Mei] and also treated by Volkmer [V1] in a different context. In particular we will show that for each pair $(\alpha, \gamma)$, the measure $\sigma_{\theta, \phi}^{\alpha, \gamma}$ has uniformly bounded total variation for $0<\theta, \phi<\pi$. Moreover, the exact range of $(\alpha, \gamma)$ will be given for which the measures are nonnegative. Finally, it turns out that they are absolutely continuous with respect to Lebesgue measure if and only if $\alpha>-1 / 2$. The method of proof employs a partial differential equation technique based on Riemann's integration method for solving Cauchy problems. These ideas were used in a recent paper [CMS] to give, among other things, a new proof of the product formula for the ultraspherical polynomials; see also the previous work by one of us, where the techniques are extended to various other situations (cf. [M1, M2, M3], and for further references, [CMS]). The product formula (1.5) can be used to define a two-parameter family of convolution structures for expansions in spheroidal wave functions via the introduction of appropriate generalized translation operators in the sense of Levitan [Le] (cf. $\S 4)$. Hence many standard tools from harmonic analysis on locally compact abelian groups become available for the explication of the harmonic structure here. The product formula leads in a natural way to a convolution for the Borel measures on $[0, \pi], M([0, \pi])$. When the measure $\sigma_{\theta, \phi}$ in (1.5) is positive, it is possible to equip $[0, \pi]$ with a hypergroup structure.

Along the same lines, we will also derive the product formula for the radial prolate spheroidal functions in the form

$$
\mathscr{S}_{\lambda}^{\alpha, \gamma}(x) \mathscr{S}_{\lambda}^{\alpha, \gamma}(y)=\int_{0}^{\infty} \mathscr{S}_{\lambda}^{\alpha, \gamma}(\xi) d \sigma_{x, y}(\xi) \quad(0<x, y<\infty),
$$

the measures $\sigma_{x, y}=\sigma_{x, y}^{\alpha, y}$ being independent of $\lambda \in[0, \infty)$. (This is not the same $\sigma$ as in (1.5), but context removes any danger of ambiguity.) It should be remarked here, that unlike the angular prolate and oblate cases, the product formula in the radial oblate case behaves somewhat differently from that in the radial prolate case, (1.6). We have some interesting results here too, which will be given elsewhere. The remarks about generalized translation, convolution, and hypergroups apply here also.

In [CMS] the convolution structures associated with the eigenfunctions of the perturbed ultraspherical equation, $u^{\prime \prime}+(2 \alpha+1)(\operatorname{ctn} \theta) u^{\prime}+[\lambda-q(\theta)] u=0 \quad(\alpha>$ $-1 / 2)$ are studied. Since the potential function $q(\theta)=\gamma \sin ^{2} \theta$ is symmetric and real analytic on $[0, \pi]$ as required in [CMS, Theorem 1.2] the existence and uniqueness of the product formula (1.5) as well as its positivity in the prolate case $\gamma \leq 0$ follow. In this article we obtain explicit and simple representations for the measures $\sigma_{\theta, \phi}^{\alpha, \gamma}$ from which the positivity for certain $\gamma>0$ can also be determined, and which furnish additional hypergroup structures of the Jacobi type beyond those described in [CS3]. The availability of a hypergroup structure 
allows a more refined study of various questions of harmonic analysis as, e.g., multiplier criteria or maximal functions (cf. [CS1, CS2]).

In addition to being useful in the harmonic analysis of eigenfunction expansions, the product formulas of Theorems 1 and 2 give interesting information about the eigenfunctions themselves. This "special functions" aspect becomes apparent when observing that the equations (1.3) and (1.4) are singular differential equations not belonging to the hypergeometric class. In fact, if they are thought of as equations in the complex plane (after substituting $z=\cos ^{2}(\theta / 2)$ and $z=\cosh ^{2}(x / 2)$, respectively), then according to the Klein-Bôcher-Ince classification [I, Chapter XX] they have two finite regular singular points at $z=0$ and $z=1$ and an irregular singular point of second species at $z=\infty$. Actually, the two equations are the same, but the product formulas range over different intervals, namely $(0,1)$ and $(1, \infty)$, respectively. This is an equation of the confluent Heun type $\left[0,2,1_{2}\right]$ (which may be reduced by a quadratic transformation to the type $[1,1,1]$, the singularities being elementary, regular, and irregular of the first species respectively). Hence the solutions lie in the "land beyond Bessel" [A2] and cannot, in general, be represented in closed form.

The remarkable fact is that it is still possible to write down the product formula in closed form because of the Riemann function techniques even in cases where there is no explicit representation of the eigenfunctions. So one way of looking at formula (1.5), for instance, is to regard it as an integral equation for the unknown function $S_{n}^{\alpha, \gamma}(\theta)$ after an appropriate choice of the variable $\phi$ as, e.g., $\phi=\pi / 2$ (cf. [V1] for consequences of this procedure in the Mathieu case). Moreover, the formulas can be used to derive uniform and pointwise estimates of the angular or radial spheroidal wave functions (cf. Theorems 3 and 4).

The spheroidal wave functions provide a rich family of examples in which to study singular differential equations and the harmonic analysis of the corresponding eigenfunction expansions. Though not in the field of classical special function theory and, in particular, not of orthogonal polynomial type, they nevertheless show a structure depending on two parameters $\alpha$ and $\gamma$, which allows distinct eigenfunction expansions to be organized into a scale or continuum of expansions [CS1], and the properties of the individual eigenfunction systems can be described by theorems about the parameters. The spheroidal wave functions thus provide a convenient test for general theorems about eigenfunction expansions.

Finally, we mention that these functions are important for various applications: they arise, for instance in signal theory where they occur naturally in the analysis of band limited signals, see, e.g., [S, P]. We intend to study the application of the positive convolution to these problems at another time. Further references can be found in [D].

\section{Product formulas for ANGUlar SPHEROIDAL WAVE FUNCTIONS}

We begin with a few properties of the hypergeometric functions. Let $(x)_{0}=$ 1 , and $(x)_{n}=x(x+1)(x+2) \cdots(x+n-1)$ for $n \in \mathbb{N}$, and let

$$
F(a, b ; c ; z)={ }_{2} F_{1}(a, b ; c ; z)=\sum_{n \in \mathbb{N}_{0}} \frac{(a)_{n}(b)_{n}}{(c)_{n} n !} z^{n} \text {. }
$$


Then one has

$$
\begin{gathered}
F(a, b ; c ; 1)=\frac{\Gamma(c) \Gamma(c-a-b)}{\Gamma(c-a) \Gamma(c-b)} \quad(\operatorname{Re} c>\operatorname{Re}(a+b)) \\
\frac{d}{d z} F(a, b ; c ; z)=\frac{a b}{c} F(a+1, b+1 ; c+1 ; z), \\
F(a, b ; c ; z)=(1-z)^{-a} F\left(a, c-b ; c ; \frac{z}{z-1}\right) \\
=(1-z)^{-b} F\left(c-a, b ; c ; \frac{z}{z-1}\right) \\
=(1-z)^{c-a-b} F(c-a, c-b ; c ; z) .
\end{gathered}
$$

Our first aim is to establish the product formula for the angular spheroidal wave functions $S_{n}^{\alpha, \gamma}(\theta), n \in \mathbb{N}_{0}, 0<\theta<\pi$. To this end we recall the following formulas for the ultraspherical polynomials:

$$
\begin{aligned}
S_{n}^{\alpha, 0}(\theta) & =P_{n}^{(\alpha+1 / 2)}(\cos \theta) / P_{n}^{(\alpha+1 / 2)}(1) \\
& =F\left(-n, n+2 \alpha+1 ; \alpha+1 ; \sin ^{2}(\theta / 2)\right)
\end{aligned}
$$

and (see [CMS, (1.8) and (1.9)])

$$
S_{n}^{\alpha, 0}(\theta) S_{n}^{\alpha, 0}(\phi)=\int_{|\theta-\phi|}^{\pi-|\theta+\phi-\pi|} S_{n}^{\alpha, 0}(\xi) K^{\alpha}(\xi, \theta, \phi) w^{\alpha}(\xi) d \xi
$$

$$
(0<\theta, \phi<\pi)
$$

with

$$
\begin{gathered}
w^{\alpha}(\xi)=\sin ^{2 \alpha+1} \xi, \\
K^{\alpha}(\xi, \theta, \phi)= \begin{cases}\frac{\Gamma(\alpha+1)}{\Gamma(\alpha+1 / 2) \Gamma(1 / 2)} \frac{\left(\psi_{0}\right)^{\alpha-1 / 2}}{\omega_{0}^{2 \alpha}} & \text { if } \xi \in(|\theta-\phi|, \pi-|\theta+\phi-\pi|), \\
0 & \text { if } \xi \notin(|\theta-\phi|, \pi-|\theta+\phi-\pi|),\end{cases}
\end{gathered}
$$

where

$$
\begin{aligned}
\psi_{0} & =[\cos (\theta-\phi)-\cos \xi][\cos \xi-\cos (\theta+\phi)], \\
\omega_{0} & =(\sin \xi)(\sin \theta)(\sin \phi) .
\end{aligned}
$$

Let $J_{\nu}$ denote the Bessel function of the first kind of order $\nu$, so that

$$
\mathscr{J}_{\nu}(z)=2^{\nu} \Gamma(\nu+1) J_{\nu}(z) / z^{\nu}=\sum_{k=0}^{\infty} \frac{\left(-z^{2} / 4\right)^{k}}{(\nu+1)_{k} k !}
$$

is an entire function that satisfies $\mathscr{J}_{\nu}(0)=1$ and $\mathscr{I}_{\nu}(x)=\mathscr{J}_{\nu}(i x)>0$ if $x \in \mathbb{R}$. Let $j_{\nu}$ be the smallest positive zero of $J_{\nu}$. For detailed information on $j_{\nu}$ such as bounds, monotonicity, and asymptotic behavior as $\nu \rightarrow-1$, see [IM] and the references cited there.

Theorem 1. Let $\alpha \geq-1 / 2, \gamma \in \mathbb{R}, 0<\theta, \phi<\pi$, and $n \in \mathbb{N}_{0}$.

(i) For $\alpha>-1 / 2$, the angular spheroidal wave functions satisfy

$$
S_{n}^{\alpha, \gamma}(\theta) S_{n}^{\alpha, \gamma}(\phi)=\int_{|\theta-\phi|}^{\pi-|\theta+\phi-\pi|} S_{n}^{\alpha, \gamma}(\xi) \mathscr{K}^{\alpha, \gamma}(\xi, \theta, \phi) w^{\alpha}(\xi) d \xi
$$


where

$$
\mathscr{K}^{\alpha, \gamma}(\xi, \theta, \phi)=K^{\alpha}(\xi, \theta, \phi) \mathscr{J}_{\alpha-1 / 2}\left(\sqrt{\gamma \psi_{0}}\right) .
$$

(ii) In the particular case of the Mathieu functions, $\alpha=-1 / 2$, one has

$$
\begin{aligned}
S_{n}^{-1 / 2, \gamma}(\theta) S_{n}^{-1 / 2, \gamma}(\phi)= & \frac{1}{2} S_{n}^{-1 / 2, \gamma}(|\theta-\phi|)+\frac{1}{2} S_{n}^{-1 / 2, \gamma}(\pi-|\theta+\phi-\pi|) \\
& +\int_{|\theta-\phi|}^{\pi-|\theta+\phi-\pi|} S_{n}^{-1 / 2, \gamma}(\xi) \mathscr{K}^{-1 / 2, \gamma}(\xi, \theta, \phi) d \xi
\end{aligned}
$$

where

(2.9)

$$
\mathscr{K}^{-1 / 2, \gamma}(\xi, \theta, \phi)=\left\{\begin{array}{l}
-\frac{1}{4} \gamma \omega_{0} \mathscr{J}_{1}\left(\sqrt{\gamma \psi_{0}}\right) \text { if } \xi \in(|\theta-\phi|, \pi-|\theta+\phi-\pi|), \\
0 \text { if } \xi \notin(|\theta-\phi|, \pi-|\theta+\phi-\pi|) .
\end{array}\right.
$$

(iii) If $\alpha>-1 / 2$ and $-\infty<\gamma \leq j_{\alpha-1 / 2}^{2}$, or $\alpha=-1 / 2$ and $\gamma \leq 0$, then $\mathscr{K}^{\alpha, \gamma}(\xi, \theta, \phi)$ is positive, symmetric in $\xi, \theta$, and $\phi$ and as a function of $\xi$, it is supported in $|\theta-\phi|<\xi<\pi-|\theta+\phi-\pi|$.

(iv) If $\gamma \geq 0$ and $\alpha \geq 0$, then

$$
\int_{|\theta-\phi|}^{\pi-|\theta+\phi-\pi|}\left|\mathscr{K}^{\alpha, \gamma}(\xi, \theta, \phi)\right| w^{\alpha}(\xi) d \xi \leq 1 .
$$

To prove Theorem 1 we will proceed (as in the ultraspherical case [CMS, §2]) from the fact that the product on the left-hand side of (2.6) (and of (2.8)) can be considered as the unique solution of a hyperbolic initial value problem which can be solved in terms of the initial function by Riemann's integration method. Set

$$
u(\xi, \eta)=u(\eta, \xi)=S_{n}^{\alpha, \gamma}(\xi) S_{n}^{\alpha, \gamma}(\eta)
$$

and observe that because of the symmetry

$$
S_{n}^{\alpha, \gamma}(\pi-\xi)=(-1)^{n} S_{n}^{\alpha, \gamma}(\xi),
$$

we may restrict our attention to $(\xi, \eta)$ for which $0 \leq \eta \leq \xi \leq \pi-\eta$. Denote this triangle by $E$. Then, since $S_{n}^{\alpha, \gamma}(\xi)$ satisfies equation (1.3) together with $S^{\prime}(0)=S^{\prime}(\pi)=0$ and $S(0)=1$ one has

$$
\begin{gathered}
\left(L_{\xi}^{\alpha, \gamma}-L_{\eta}^{\alpha, \gamma}\right) u(\xi, \eta)=0, \quad((\xi, \eta) \in E), \\
u(\xi, 0)=S_{n}^{\alpha, \gamma}(\xi), \quad u_{\eta}(\xi, 0)=0, \quad(0<\xi<\pi),
\end{gathered}
$$

where

$$
L_{\xi}^{\alpha, \gamma}=d^{2} / d \xi^{2}+(2 \alpha+1)(\cot \xi) d / d \xi-\gamma \sin ^{2} \xi .
$$

The solution of (2.10) requires explicit knowledge of the corresponding Riemann function. For any fixed point $(\theta, \phi) \in E$ let $\Delta_{\theta, \phi}$ be the characteristic triangle with vertices $P=(\theta, \phi), Q_{0}$, and $R_{0}$, where $Q_{\varepsilon}=(\theta-\phi+\varepsilon, \varepsilon)$ and $R_{\varepsilon}=(\theta+\phi-\varepsilon, \varepsilon)$. Then for $(\xi, \eta) \in \Delta_{\theta, \phi}$, the Riemann function is defined as the unique solution $v(\xi, \eta)=v(\xi, \eta ; \theta, \phi)=R^{\alpha, \gamma}(\xi, \eta ; \theta, \phi)$ of the characteristic boundary value problem: 


$$
\begin{aligned}
& \left(L_{\xi}^{\alpha, \gamma}-L_{\eta}^{\alpha, \gamma^{*}}\right) v(\xi, \eta)=0 \quad\left((\xi, \eta) \in \Delta_{\theta, \phi}\right), \\
& v_{\xi}+v_{\eta}-\left(\frac{\alpha+1 / 2}{\tan \xi}+\frac{\alpha+1 / 2}{\tan \eta}\right) v=0 \quad(\xi-\eta=\theta-\phi), \\
& v_{\xi}-v_{\eta}-\left(\frac{\alpha+1 / 2}{\tan \xi}-\frac{\alpha+1 / 2}{\tan \eta}\right) v=0 \quad(\xi+\eta=\theta+\phi), \\
& v(\theta, \phi)=1
\end{aligned}
$$

(the asterisk $(*)$ denotes the adjoint operator). Let

$$
\begin{gathered}
\chi=\chi(\xi, \eta ; \theta, \phi)=[\cos (\theta-\phi)-\cos (\xi-\eta)][\cos (\xi+\eta)-\cos (\theta+\phi)], \\
\psi=\psi(\xi, \eta ; \theta, \phi)=[\cos (\theta-\phi)-\cos (\xi+\eta)][\cos (\xi-\eta)-\cos (\theta+\phi)], \\
\omega=4(\sin \xi)(\sin \eta)(\sin \theta)(\sin \phi) \\
W=\frac{-\chi}{\omega}, \quad \Phi=\frac{W}{W-1}=\frac{\chi}{\psi} \\
\mathscr{R}^{\alpha}(\xi, \eta ; \theta, \phi)=\left(\frac{(\sin \xi)(\sin \eta)}{(\sin \theta)(\sin \phi)}\right)^{\alpha+1 / 2}\left(\frac{\psi}{\omega}\right)^{|\alpha|-1 / 2}
\end{gathered}
$$

Lemma. For $\alpha \geq-1 / 2, \gamma \in \mathbb{R}$, and $(\xi, \eta) \in \Delta_{\theta, \phi}$,

(2.12)

$$
\begin{aligned}
R^{\alpha, \gamma} & (\xi, \eta ; \theta, \phi) \\
& =\mathscr{R}^{\alpha}(\xi, \eta ; \theta, \phi) \sum_{k \in \mathbb{N}_{0}} F(k+1 / 2-|\alpha|, 1 / 2-|\alpha| ; k+1 ; \Phi) \frac{(-\gamma \chi / 4)^{k}}{(k !)^{2}}
\end{aligned}
$$

in particular, for $\alpha=-1 / 2$

$$
R^{-1 / 2, \gamma}(\xi, \eta ; \theta, \phi)=J_{0}(\sqrt{\gamma \chi}) .
$$

(Notice that in the ultraspherical case $\gamma=0$, (2.12) collapses to the $k=0$ term to yield $R^{\alpha, 0}(\xi, \eta ; \theta, \phi)=\mathscr{R}^{\alpha}(\xi, \eta ; \theta, \phi) F(1 / 2-|\alpha|, 1 / 2-|\alpha| ; 1 ; \Phi)$.)

Proof. The substitution of the independent and dependent variables

$$
\begin{gathered}
X_{0}=\cos (\theta+\phi), \quad Y_{0}=\cos (\theta-\phi) \\
X=\cos (\xi+\eta), \quad Y=\cos (\xi-\eta) \\
v(\xi, \eta ; \theta, \phi)=\left(\frac{(\sin \xi)(\sin \eta)}{(\sin \theta)(\sin \phi)}\right)^{\alpha+1 / 2} V\left(X, Y ; X_{0}, Y_{0}\right)
\end{gathered}
$$

leads to the selfadjoint "normal" form of problem (2.11),

$$
\begin{gathered}
V_{X Y}+\left(\frac{\alpha^{2}-1 / 4}{(X-Y)^{2}}-\frac{\gamma}{4}\right) V=0 \quad\left(X_{0}<X<Y<Y_{0}\right), \\
V\left(X_{0}, Y ; X_{0}, Y_{0}\right)=V\left(X, Y_{0} ; X_{0}, Y_{0}\right)=1 .
\end{gathered}
$$

Its solution was given by Henrici [H], (cf. also [L]) as a confluent hypergeometric function of two auxiliary variables

$$
W_{1}=\left(X-X_{0}\right)\left(Y-Y_{0}\right)
$$




$$
W_{2}=\frac{\left(X-X_{0}\right)\left(Y-Y_{0}\right)}{(X-Y)\left(X_{0}-Y_{0}\right)}
$$

namely

$$
\begin{aligned}
V\left(X, Y ; X_{0}, Y_{0}\right) & =\Xi_{2}\left(1 / 2+\alpha, 1 / 2-\alpha ; 1 ; W_{2}, \gamma W_{1} / 4\right) \\
& =\sum_{k, n \in \mathbb{N}_{0}} \frac{(1 / 2+\alpha)_{n}(1 / 2-\alpha)_{n}}{k ! n !(k+n) !} W_{2}^{n}\left(\gamma W_{1} / 4\right)^{k}
\end{aligned}
$$

which converges for $\left|W_{2}\right|<1$ (for properties of hypergeometric functions of several variables cf., e.g., [E, §5]). Writing the inner sum on the right of $(2.15)$ as an ordinary hypergeometric function and extending it analytically to the halfplane $\operatorname{Re} W_{2}<1 / 2$ by (2.2), (2.3), one arrives at

$$
\begin{aligned}
V\left(X, Y ; X_{0}, Y_{0}\right) & =\left(1-W_{2}\right)^{|\alpha|-1 / 2} \\
& \times \sum_{k \in \mathbb{N}_{0}} F\left(k+1 / 2-|\alpha|, 1 / 2-|\alpha| ; k+1 ; \frac{W_{2}}{W_{2}-1}\right) \frac{\left(\gamma W_{1} / 4\right)^{k}}{(k !)^{2}}
\end{aligned}
$$

which is valid for all $X_{0}<X<Y<Y_{0}$. Returning to the original variables $\xi, \eta, \theta, \phi$ via (2.13) and observing that $W_{2}=W, W_{1}=-\chi$ finally yields the lemma. Notice that in the special case $\alpha=-1 / 2$, the boundary value problem (2.11) reduces to that for the well-known telegraph equation which was solved by Riemann already in terms of the Bessel function $J_{0}$ (cf., e.g., [G, (4.84)] or [L]).

Remark. In the proofs of the product formulas for the Bessel functions and for the Laguerre functions given in [M1], extensive use was made of the fact that after transformation to a selfadjoint normal form, the characteristic boundary value problems defining the corresponding Riemann functions are exactly of the form (2.14), with $\gamma=0$ in the Bessel case and $\gamma=$ constant $\neq 0$ in the Laguerre case. This explains the formal similarity of the product formulas for the Laguerre and spheroidal wave functions (though being defined on different intervals). In particular, one can say that the relationship between the Laguerre and Bessel cases is the same as between the spheroidal and ultraspherical cases.

Proof of Theorem 1. (i) We discuss here the case $-1 / 2<\alpha \leq 0$; the case $\alpha>0$ is dealt with by similar arguments. The argument used in [CMS, Theorem 1.2] in the ultraspherical case yields (2.6) with $\mathscr{K}^{\alpha, \gamma}$ obtained from the Riemann function (2.12) by

$$
\begin{aligned}
\mathscr{K}^{\alpha, \gamma}(\xi, \theta, \phi) & =\lim _{\eta \rightarrow 0+}\left\{-\frac{1}{2}(\sin \eta)^{2 \alpha+1} \frac{\partial}{\partial \eta}\left[(\sin \xi \sin \eta)^{-2 \alpha-1} R^{\alpha, \gamma}(\xi, \eta ; \theta, \phi)\right]\right\} \\
= & -2^{2 \alpha} \lim _{\eta \rightarrow 0+}\left\{(\sin \eta)^{2 \alpha+1}\right. \\
& \left.\times \frac{\partial}{\partial \eta}\left[\psi^{-\alpha-1 / 2} \sum_{k \in \mathbb{N}_{0}} F(k+\alpha+1 / 2, \alpha+1 / 2 ; k+1 ; \Phi) \frac{(-\gamma \chi / 4)^{k}}{(k !)^{2}}\right]\right\} .
\end{aligned}
$$


In view of $(2.1)$, one has

$$
\begin{aligned}
& \frac{\partial}{\partial \eta}\left[\psi^{-\alpha-1 / 2} \sum_{k \in \mathbb{N}_{0}} F(k+\alpha+1 / 2, \alpha+1 / 2 ; k+1 ; \Phi) \frac{(-\gamma \chi / 4)^{k}}{(k !)^{2}}\right] \\
&=-(\alpha+1 / 2) \psi^{-\alpha-3 / 2} \frac{\partial \psi}{\partial \eta} \sum_{k \in \mathbb{N}_{0}} F(k+\alpha+1 / 2, \alpha+1 / 2 ; k+1 ; \Phi) \frac{(-\gamma \chi / 4)^{k}}{(k !)^{2}} \\
& \quad+\psi^{-\alpha-1 / 2} \sum_{k \in \mathbb{N}_{0}} \frac{(k+\alpha+1 / 2)(\alpha+1 / 2)}{k+1} \\
& \quad \times F(k+\alpha+3 / 2, \alpha+3 / 2 ; k+2 ; \Phi) \frac{\partial \Phi}{\partial \eta} \frac{(-\gamma \chi / 4)^{k}}{(k !)^{2}} \\
& \quad+\psi^{-\alpha-1 / 2} \sum_{k \in \mathbb{N}} F(k+\alpha+1 / 2, \alpha+1 / 2 ; k+1 ; \Phi) \frac{(-\gamma \chi / 4)^{k}}{(k-1) ! k !}\left(-\frac{\gamma}{4} \frac{\partial \chi}{\partial \eta}\right) \\
&= S_{1}+S_{2}+S_{3} .
\end{aligned}
$$

The term-by-term differentiation is justified because all three series converge uniformly. We show this first for $\alpha<0$. Observe that $0 \leq \Phi<1$ if $\eta \in$ $(0, \pi / 2]$, so that for some constants $A_{1}$ and $A_{2}$,

$$
\begin{aligned}
0 & \leq F(k+\alpha+1 / 2, \alpha+1 / 2 ; k+1 ; \Phi) \\
& \leq F(k+\alpha+1 / 2, \alpha+1 / 2 ; k+1 ; 1) \\
& =\frac{\Gamma(k+1) \Gamma(-2 \alpha)}{\Gamma(1 / 2-\alpha) \Gamma(k+1 / 2-\alpha)} \leq A_{1} k^{\alpha+1 / 2}
\end{aligned}
$$

and from (2.4)

$$
\begin{aligned}
\frac{(k+\alpha+1 / 2)(\alpha+1 / 2)}{k+1} F(k+\alpha+3 / 2, \alpha+3 / 2 ; k+2 ; \Phi)(1-\Phi)^{2 \alpha+1} \\
\quad=\frac{(k+\alpha+1 / 2)(\alpha+1 / 2)}{k+1} F(1 / 2-\alpha, k+1 / 2-\alpha ; k+2 ; \Phi) \\
\quad \leq \frac{(k+\alpha+1 / 2)(\alpha+1 / 2)}{k+1} F(1 / 2-\alpha, k+1 / 2-\alpha ; k+2 ; 1) \\
\quad=\frac{\Gamma(k+1) \Gamma(2 \alpha+1)}{\Gamma(k+\alpha+1 / 2) \Gamma(\alpha+1 / 2)} \\
\leq A_{2} k^{1 / 2-\alpha}
\end{aligned}
$$

If $\alpha=0$, a very similar argument is based on the estimate

$$
F(k+1 / 2,1 / 2 ; k+1 ; z) \leq 1-\frac{\Gamma(k+1)}{\Gamma(k+1 / 2) \Gamma(1 / 2)} \log (1-z) .
$$

Now,

$$
\frac{\partial \psi}{\partial \eta} \rightarrow 2 \omega_{0}, \quad \frac{\partial \chi}{\partial \eta} \rightarrow-2 \omega_{0}, \quad \frac{\partial \Phi}{\partial \eta} \rightarrow-4 \omega_{0} / \psi_{0} \quad(\eta \rightarrow 0+)
$$

and so remain bounded in the limit for any $\theta-\phi<\xi<\theta+\phi$. Since, moreover, $1 / \psi \leq 1 / \psi_{0}$, it follows that $S_{1}$ and $S_{3}$ are bounded as $\eta \rightarrow 0+$, and

$$
\mathscr{K}^{\alpha, \gamma}(\xi, \theta, \phi)=-2^{2 \alpha} \lim _{\eta \rightarrow 0+}\left\{(\sin \eta)^{2 \alpha+1} S_{2}(\xi, \eta ; \theta, \phi)\right\} \text {. }
$$


In view of (2.16) and the fact that

$$
\lim _{\eta \rightarrow 0+}(\sin \eta)^{2 \alpha+1} \psi^{-\alpha-1 / 2}(1-\Phi)^{-2 \alpha-1}=\frac{\psi_{0}^{\alpha+1 / 2}}{\left(4 \omega_{0}\right)^{2 \alpha+1}},
$$

the kernel can now be calculated to be

$$
\begin{aligned}
\mathscr{K}^{\alpha, \gamma}(\xi, \theta, \phi) & =2^{2 \alpha} \frac{\psi_{0}^{\alpha-1 / 2}}{\left(4 \omega_{0}\right)^{2 \alpha}} \sum_{k \in \mathbb{N}_{0}} \frac{\Gamma(k+1) \Gamma(2 \alpha+1)}{\Gamma(k+\alpha+1 / 2) \Gamma(\alpha+1 / 2)} \frac{\left(-\gamma \psi_{0} / 4\right)^{k}}{(k !)^{2}} \\
& =\frac{\psi_{0}^{\alpha-1 / 2}}{\left(2 \omega_{0}\right)^{2 \alpha}} \frac{\Gamma(2 \alpha+1)}{\Gamma^{2}(\alpha+1 / 2)} \mathscr{J}_{\alpha-1 / 2}\left(\sqrt{\gamma \psi_{0}}\right) .
\end{aligned}
$$

Thus using the Legendre's duplication formula for the gamma function we obtain (2.7).

(ii) In the special case $\alpha=-1 / 2$ we have for $0<\varepsilon<\pi / 2$, (cf. [CMS, equation (3.9)])

$$
\begin{aligned}
u(\theta, \phi)= & \frac{1}{2}\left(\left.(u v)\right|_{Q_{\varepsilon}}+\left.(u v)\right|_{R_{\varepsilon}}\right) \\
& +\left.\frac{1}{2} \int_{\theta-\phi+\varepsilon}^{\theta+\phi-\varepsilon}\left[u_{\eta}(\xi, \eta) v(\xi, \eta)\right]\right|_{\eta=\varepsilon} d \xi \\
& -\left.\frac{1}{2} \int_{\theta-\phi-\varepsilon}^{\theta+\phi-\varepsilon}\left[u(\xi, \eta) v_{\eta}(\xi, \eta)\right]\right|_{\eta=\varepsilon} d \xi \\
= & T_{1}(\varepsilon)+T_{2}(\varepsilon)+T_{3}(\varepsilon),
\end{aligned}
$$

with $v(\xi, \eta)=v(\xi, \eta ; \theta, \phi)=J_{0}(\sqrt{\gamma \chi})$.

Now, $\lim _{\varepsilon \rightarrow 0+} v\left(Q_{\varepsilon}\right)=\lim _{\varepsilon \rightarrow 0+} v\left(R_{\varepsilon}\right)=1$, since $J_{0}(0)=1$, so

$$
\lim _{\varepsilon \rightarrow 0+} T_{1}(\varepsilon)=\frac{1}{2}[u(\theta-\phi)+u(\theta+\phi)] .
$$

Since $v(\xi, \eta)$ is uniformly bounded and $u_{\eta}(\varepsilon) \rightarrow 0$ as $\varepsilon \rightarrow 0+, \lim _{\varepsilon \rightarrow 0+} T_{2}(\varepsilon)$ $=0$. Finally

$$
v_{\eta}(\xi, \eta)=-\frac{\gamma}{4} \mathscr{J}_{1}(\sqrt{\gamma \chi}) \frac{\partial \chi}{\partial \eta}
$$

and so

$$
\mathscr{K}^{-1 / 2, \gamma}(\xi, \theta, \phi)=\lim _{\varepsilon \rightarrow 0+}\left[-\frac{1}{2} v_{\eta}(\xi, \eta)\right]=-\frac{\gamma}{4} \omega_{0} \mathscr{F}_{1}\left(\sqrt{\gamma \psi_{0}}\right) .
$$

This yields (2.9).

(iii) The general theory [CMS, Theorem $1.2($ iv)] predicts the positivity of $\mathscr{K}^{\alpha, \gamma}$ when $\alpha>-1 / 2$ and $\gamma \leq 0$, for in that case $\gamma \sin ^{2} \theta$ is a nonincreasing function on $0 \leq \theta \leq \pi / 2$. The positivity is also apparent from the explicit formulas (2.7) and (2.9) since if $\gamma \leq 0$ and $\nu=\alpha-1 / 2, \mathscr{J}_{\nu}\left(\sqrt{\gamma \psi_{0}}\right)=$ $\mathscr{F}_{\nu}\left(\sqrt{|\gamma| \psi_{0}}\right) \geq 0$, while if $0 \leq \gamma \leq j_{\nu}^{2}$, then $\sqrt{\gamma \psi_{0}} \leq j_{\nu}$, and so $\mathscr{J}_{\nu}\left(\sqrt{\gamma \psi_{0}}\right) \geq 0$. Symmetry follows since $\omega_{0}$ and $\psi_{0}$ are symmetric in the three variables.

(iv) If $\alpha \geq 0$, then $\nu=\alpha-1 / 2 \geq-1 / 2$, so $\mathscr{J}_{\nu}(x)$ is a character for a hypergroup. (See, for instance, the product formula for $\mathscr{J}_{\nu}$ given in [Sc, equations (1.1)-(1.3), which is in turn based on a theorem of Gegenbauer [W, 
p. 367].) Hence $\left|\mathscr{J}_{\nu}(x)\right| \leq\left|\mathscr{L}_{\nu}(0)\right|=1$. Thus if $\gamma \geq 0$ and $\alpha \geq 0$,

$$
\begin{aligned}
& \int_{|\theta-\phi|}^{\pi-|\theta+\phi-\pi|}\left|\mathscr{K}^{\alpha, \gamma}(\xi, \theta, \phi)\right| w^{\alpha}(\xi) d \xi \\
& \quad \leq \int_{|\theta-\phi|}^{\pi-|\theta+\phi-\pi|} K^{\alpha}(\xi, \theta, \phi) w^{\alpha}(\xi) d \xi=1
\end{aligned}
$$

(as can be seen by setting $n=0$ in (2.5)).

\section{PRODUCT Formulas for RADIAL PROLATE SPHEROIDAL WAVE FUNCTIONS}

Let us now turn to the product formula for the eigenfunctions of the SturmLiouville equation (1.4), the radial prolate spheroidal wave functions $\mathscr{S}_{\lambda}^{\alpha, \gamma}(x)$, $\lambda, x \geq 0$. In the following we use the same notations $\chi, \psi, \omega, \mathscr{K}$ as before, but with the new meaning (indicated sometimes by $x, y$ instead of $\theta, \phi$ )

$$
\begin{gathered}
\chi=\chi(\xi, \eta ; x, y)=[\cosh (x-y)-\cosh (\xi-\eta)][\cosh (\xi+\eta)-\cosh (x+y)], \\
\psi=\psi(\xi, \eta ; x, y)=[\cosh (x-y)-\cosh (\xi+\eta)][\cosh (\xi-\eta)-\cosh (x+y)], \\
\omega=4(\sinh \xi)(\sinh \eta)(\sinh x)(\sinh y), \\
\Phi=\frac{\chi}{\psi}, \quad \psi_{0}=\psi(\xi, 0 ; x, y), \quad \omega_{0}=(\sinh \xi)(\sinh x)(\sinh y) .
\end{gathered}
$$

Theorem 2. Let $\alpha \geq-1 / 2, \gamma \leq 0, \lambda \geq 0$ and $x, y \in(0, \infty)$.

(i) For $\alpha>-1 / 2$,

$$
\mathscr{S}_{\lambda}^{\alpha, \gamma}(x) \mathscr{S}_{\lambda}^{\alpha, \gamma}(y)=\int_{|x-y|}^{x+y} \mathscr{S}_{\lambda}^{\alpha, \gamma}(\xi) \mathscr{K}^{\alpha, \gamma}(\xi, x, y)(\sinh \xi)^{2 \alpha+1} d \xi
$$

where

$$
\mathscr{K}^{\alpha, \gamma}(\xi, x, y)=\left\{\begin{array}{c}
\frac{\Gamma(\alpha+1)}{\Gamma(\alpha+1 / 2) \Gamma(1 / 2)} \frac{\psi_{0}^{\alpha-1 / 2}}{\omega_{0}^{2 \alpha}} \mathcal{F}_{\alpha-1 / 2}\left(\sqrt{|\gamma| \psi_{0}}\right) \\
\text { if } \xi \in(|x-y|, x+y), \\
0 \quad \text { if } \xi \notin(|x-y|, x+y) .
\end{array}\right.
$$

(ii) If $\alpha=-1 / 2$,

$$
\begin{aligned}
\mathscr{S}_{\lambda}^{-1 / 2, \gamma}(x) \mathscr{S}_{\lambda}^{-1 / 2, \gamma}(y)= & \frac{1}{2} \mathscr{S}_{\lambda}^{-1 / 2, \gamma}(|x-y|)+\frac{1}{2} \mathscr{S}_{\lambda}^{-1 / 2, \gamma}(x+y) \\
& +\int_{|x-y|}^{x+y} \mathscr{S}_{\lambda}^{-1 / 2, \gamma}(\xi) \mathscr{K}^{-1 / 2, \gamma}(\xi, x, y) d \xi
\end{aligned}
$$

where

$$
\mathscr{K}^{-1 / 2, \gamma}(\xi, x, y)= \begin{cases}\frac{1}{4}|\gamma| \omega_{0} \mathscr{I}_{1}\left(\sqrt{|\gamma| \psi_{0}}\right) & \text { if } \xi \in(|x-y|, x+y), \\ 0 & \text { if } \xi \notin(|x-y|, x+y) .\end{cases}
$$

(iii) For any $0<x, y<\infty$ and $|x-y|<\xi<x+y$, the kernel $\mathscr{K}^{\alpha, \gamma}(\xi, x, y)$ is positive and symmetric in $\xi, x$, and $y$. 
Proof. The proof follows the same lines as the proof of Theorem 1. The characteristic boundary value problem for the Riemann function now reads

$$
\begin{aligned}
& v_{\xi \xi}-v_{\eta \eta}-\left(\frac{2 \alpha+1}{\tanh \xi} v\right)_{\xi}+\left(\frac{2 \alpha+1}{\tanh \eta} v\right)_{\eta} \\
& \quad-\gamma\left[\sinh ^{2} \xi-\sinh ^{2} \eta\right] v=0 \quad\left((\xi, \eta) \in \Delta_{x, y}\right), \\
& v_{\xi}+v_{\eta}-\left(\frac{\alpha+1 / 2}{\tanh \xi}+\frac{\alpha+1 / 2}{\tanh \eta}\right) v=0 \quad(\xi-\eta=x-y), \\
& v_{\xi}-v_{\eta}-\left(\frac{\alpha+1 / 2}{\tanh \xi}-\frac{\alpha+1 / 2}{\tanh \eta}\right) v=0 \quad(\xi+\eta=x+y), \\
& v(x, y)=1 .
\end{aligned}
$$

Its solution is given by

$$
\begin{aligned}
R^{\alpha, \gamma}(\xi, \eta ; x, y)= & \left(\frac{(\sinh \xi)(\sinh \eta)}{(\sinh x)(\sinh y)}\right)^{\alpha+1 / 2}\left(\frac{\psi}{\omega}\right)^{|\alpha|-1 / 2} \\
& \times \sum_{k \in \mathbb{N}_{0}} F(k+1 / 2-|\alpha|, 1 / 2-|\alpha| ; k+1 ; \Phi) \frac{(-\gamma \chi / 4)^{k}}{(k !)^{2}} .
\end{aligned}
$$

This follows immediately from the fact that the transformations

$$
\begin{aligned}
X_{0}=\cosh (x+y), \quad Y_{0} & =\cosh (x-y), \quad X=\cosh (\xi+\eta), \quad Y=\cosh (\xi-\eta), \\
v(\xi, \eta ; x, y) & =\left(\frac{(\sinh \xi)(\sinh \eta)}{(\sinh x)(\sinh y)}\right)^{\alpha+1 / 2} V\left(X, Y ; X_{0}, Y_{0}\right)
\end{aligned}
$$

reduce problem (3.1) to the same selfadjoint problem (2.14) as was solved in the angular spheroidal case, but now in the domain $Y_{0}<Y<X<X_{0}$. Using the representation (2.15) of $V$, the rest of the proof is straightforward and left to the reader.

Remark. For $\gamma=0$, the results of Theorem 2 coincide with the product formula for ultraspherical functions due to Flensted-Jensen and Koornwinder [FK] (cf. also $[\mathrm{K}]$ and, for a partial differential equation approach, [M3]).

\section{Convolution STRUCTURES, HYPERGROUPS, AND INEQUALITIES}

The measure $\sigma_{\theta, \phi}^{\alpha, \gamma}$ of $(1.5)$ is defined implicitly by Theorem 1 and is given by

$$
\begin{aligned}
d \sigma_{\theta, \phi}^{\alpha, \gamma}(\xi) & =\mathscr{K}^{\alpha, \gamma}(\xi, \theta, \phi) w^{\alpha}(\xi) d \xi \quad(\alpha>-1 / 2, \gamma \in \mathbb{R}), \\
d \sigma_{\theta, \phi}^{-1 / 2, \gamma}(\xi) & =\frac{1}{2} d \delta_{|\theta-\phi|}(\xi)+\frac{1}{2} d \delta_{\pi-|\theta+\phi-\pi|}(\xi)+\mathscr{K}^{-1 / 2, \gamma}(\xi, \theta, \phi) d \xi
\end{aligned}
$$

where $\delta_{t}$ denotes the unit point mass concentrated at $t$. This gives rise to two products or convolutions on $M([0, \pi])$ which are continuous with respect to the total variation norm $\|\cdot\|$. If $\mu$ and $\nu$ belong to $M([0, \pi])$, the first product $\mu \# \nu=\mu \#^{\alpha, \gamma} \nu$, is defined by its action on a continuous function $f$ :

$$
\int_{0}^{\pi} f(s) d(\mu \# \nu)(s)=\int_{0}^{\pi} \int_{0}^{\pi} \int_{0}^{\pi} f(\xi) d \sigma_{\theta, \phi}^{\alpha, \gamma}(\xi) d \mu(\theta) d \nu(\phi) .
$$


The explicit form of $\sigma_{\theta, \phi}^{\alpha, \gamma}$ given in (4.1) shows that the innermost integral is a measurable function of $(\theta, \phi)$. The product \# is a continuous operation on $M([0, \pi])$ provided that $A^{\alpha, \gamma}=\sup \left\{\left\|\sigma_{\theta}, \phi\right\|: 0 \leq \theta, \phi \leq \pi\right\}<\infty$. In fact, we have the inequality $\|\mu \# \nu\| \leq A^{\alpha, \gamma}\|\mu\|\|\nu\|$. Let $\mu_{n}^{\wedge}=\int_{0}^{\pi} S_{n}^{\alpha, \gamma}(\xi) d \mu(\xi)$, then substituting $S_{n}^{\alpha, \gamma}$ for $f$ in (4.2) and using (1.5) we have $(\mu \# \nu)_{n}^{\wedge}=\mu_{n}^{\wedge} \nu_{n}^{\wedge}$, so the product is commutative and associative. Thus if $A^{\alpha, \gamma} \leq 1,(M([0, \pi])$, $\left.\#^{\alpha, \gamma}\right)$ is a commutative Banach algebra. There is also a convolution on an appropriate $L^{1}$ space. In particular if

$$
\|f\|_{1}=\|f\|_{1, \alpha}=\int_{0}^{\pi}|f(\xi)| w^{\alpha}(\xi) d \xi<\infty
$$

define

$$
(f \# g)(\xi)=\left(f \#^{\alpha, \gamma} g\right)(\xi)=\int_{0}^{\pi} \int_{0}^{\pi} f(\theta) g(\phi) d \sigma_{\theta, \phi}(\xi) w^{\alpha}(\theta) w^{\alpha}(\phi) d \theta d \phi
$$

and $f_{n}^{\wedge}=\int_{0}^{\pi} S_{n}^{\alpha, \gamma}(\xi) f(\xi) w^{\alpha}(\xi) d \xi$. Examination of (4.1) shows that $f \# g$ is a well-defined measurable function. The product satisfies

$$
\|f \# g\|_{1} \leq A^{\alpha, \gamma}\|f\|_{1}\|g\|_{1} \text { and }(f \# g)_{n}^{\wedge}=f_{n}^{\wedge} g_{n}^{\wedge} \text {. }
$$

The product formula also gives rise to an inequality for the spheroidal wave functions since (1.5) implies

$$
\left|S_{n}^{\alpha, \gamma}(\theta)\right|\left|S_{n}^{\alpha, \gamma}(\phi)\right| \leq A^{\alpha, \gamma}\left\|S_{n}^{\alpha, \gamma}\right\|_{\infty} \quad(0 \leq \theta, \phi \leq \pi),
$$

whence $\left\|S_{n}^{\alpha, \gamma}\right\|_{\infty} \leq A^{\alpha, \gamma}$.

The second kind of convolution is based on the ideas in [CMS, $\S 4$ ] and uses a renormalized measure defined by

$$
d \tau_{\theta, \phi}^{\alpha, \gamma}(\xi)=S_{0}^{\alpha, \gamma}(\xi)\left(S_{0}^{\alpha, \gamma}(\theta) S_{0}^{\alpha, \gamma}(\phi)\right)^{-1} d \sigma_{\theta, \phi}^{\alpha, \gamma}(\xi) .
$$

Notice that $S_{0}^{\alpha, \gamma}(\xi)$ is positive throughout $[0, \pi]$ because $S_{0}^{\alpha, \gamma}(0)=S_{0}^{\alpha, \gamma}(\pi)=$ 1 and has no zeros in the interval. So if $R_{k}^{\alpha, \gamma}(\xi)=S_{k}^{\alpha, \gamma}(\xi) / S_{0}^{\alpha, \gamma}(\xi)$ we have the product formula

$$
R_{n}^{\alpha, \gamma}(\theta) R_{n}^{\alpha, \gamma}(\phi)=\int_{0}^{\pi} R_{n}^{\alpha, \gamma}(\xi) d \tau_{\theta, \phi}^{\alpha, \gamma}(\xi)
$$

and the convolution $*=*^{\alpha, \gamma}$ is defined by

$$
\int_{0}^{\pi} f(\xi) d(\mu * \nu)(\xi)=\int_{0}^{\pi} \int_{0}^{\pi} \int_{0}^{\pi} f(\xi) d \tau_{\theta, \phi}^{\alpha, \gamma}(\xi) d \mu(\theta) d \nu(\phi) .
$$

This operation is also commutative and associative and defines a convolution on the space of functions $f$ for which

$$
\int_{0}^{\pi}|f(\xi)| d m^{\alpha, \gamma}(\xi)<\infty
$$

where

$$
d m^{\alpha, \gamma}(\xi)=\left[S_{0}^{\alpha, \gamma}(\xi)\right]^{2} w^{\alpha}(\xi) d \xi .
$$

If $\sigma_{\theta, \phi}^{\alpha, \gamma}$ is a positive measure for every $\theta$ and $\phi$ in $[0, \pi]$, then so is $\tau_{\theta, \phi}^{\alpha, \gamma}$. Moreover, if we set $n=0$ in (4.3), $\left\|\tau_{\theta, \phi}^{\alpha, \gamma}\right\|=1$, so $*$ is probability preserving. 
That is, if $\mu$ and $\nu$ are probability measures (positive with unit mass), then so is $\mu * \nu$. In particular we have for the unit point masses

$$
\delta_{\theta} * \delta_{\phi}=\tau_{\theta, \phi}^{\alpha, \gamma} \quad(0 \leq \theta, \phi \leq \pi) .
$$

Equation (4.3) yields the global inequality $\left\|R_{n}^{\alpha, \gamma}\right\|_{\infty}=R_{n}^{\alpha, \gamma}(0)=1$, from which we obtain the pointwise inequality

$$
\left|S_{n}^{\alpha, \gamma}(\xi)\right| \leq S_{0}^{\alpha, \gamma}(\xi) \quad(0 \leq \xi \leq \pi) .
$$

Finally we remark that

$$
T^{\phi} f(\theta)=\int_{0}^{\pi} f(\xi) d \tau_{\theta, \phi}^{\alpha, \gamma}(\xi)
$$

is a generalized translation in the sense of Levitan [Le]. If $f(\xi)=\sum_{n=0}^{\infty} c_{n} R_{n}^{\alpha, \gamma}(\xi)$, then $T^{\phi} f(\xi)=\sum_{n=0}^{\infty} c_{n} R_{n}^{\alpha, \gamma}(\xi) R_{n}^{\alpha, \gamma}(\phi)$, and

$$
(f * g)(\xi)=\int_{0}^{\pi} T^{\phi} f(\theta) g(\phi) d m^{\alpha, \gamma}(\phi) .
$$

We summarize these observations and give some particular bounds in

Theorem 3. (i) If $\gamma \in \mathbb{R}$ and $\alpha \geq-1 / 2$, then $M([0, \pi])$ has a continuous convolution $\#^{\alpha, \gamma}$ which satisfies $\left\|\mu \#^{\alpha, \gamma} \nu\right\| \leq A^{\alpha, \gamma}\|\mu\|\|\nu\|$ where $A^{\alpha, \gamma}=$ $\sup \left\{\left\|\sigma_{\theta, \phi}\right\|: 0 \leq \theta, \phi \leq \pi\right\}$. Moreover $\left\|S_{n}^{\alpha, \gamma}\right\|_{\infty} \leq A^{\alpha, \gamma}$, and $A^{\alpha, \gamma}$ satisfies the following:

(a) If $\gamma \geq 0$ and $\alpha \geq 0$, then $A^{\alpha, \gamma}=1$.

(b) If $\gamma \geq 0$ and $\alpha>-1 / 2$ then $A^{\alpha, \gamma} \leq \max _{0 \leq x \leq \gamma}\left|\mathscr{F}_{\alpha-1 / 2}(\sqrt{x})\right|$.

(c) If $\gamma \geq 0$, then $A^{-1 / 2, \gamma} \leq 1+\frac{\gamma}{4} \max _{0 \leq x \leq \gamma}\left|\mathscr{J}_{1}(\sqrt{x})\right|$.

(d) If $\gamma \leq 0$ and $\alpha>-1 / 2$ then $A^{\alpha, \gamma} \leq\left|\mathcal{F}_{\alpha-1 / 2}(\sqrt{|\gamma|})\right|$.

(e) If $\gamma \leq 0$, then $A^{-1 / 2, \gamma} \leq 1+\frac{\gamma}{4} \mathscr{F}_{1}(\sqrt{|\gamma|})$.

(ii) If $\gamma \geq 0$ and $\alpha \geq 0,\left(M([0, \pi]), \#^{\alpha, \gamma}\right)$ is a Banach algebra.

(iii) If $\alpha>-1 / 2$ and $-\infty<\gamma \leq j_{\alpha-1 / 2}^{2}$, or $\alpha=-1 / 2$ and $\gamma \leq 0$, then $\left([0, \pi], *^{\alpha, \gamma}\right)$ is a Jacobi type $(\alpha, \alpha)$ hypergroup with Hermitian characters $\left\{R_{n}^{\alpha, \gamma}\right\}_{n=0}^{\infty}$, identity $\delta_{0}$, and Haar measure $m^{\alpha, \gamma}$. Moreover

$$
\left|S_{n}^{\alpha, \gamma}(\xi)\right| \leq S_{0}^{\alpha, \gamma}(\xi) \quad(0 \leq \xi \leq \pi) .
$$

The reader is referred to [CS3] for a general discussion of Jacobi type hypergroups and to [CMS, §4] for a discussion of those hypergroups which arise from a general class of differential equations which includes (1.3).

Proof. (i) In light of the discussion preceding the theorem, it only remains to establish the estimates (a)-(e). Observe that if $\alpha>-1 / 2$,

$$
\begin{aligned}
\left\|\sigma_{\theta, \phi}^{\alpha, \gamma}\right\| & =\int_{|\theta-\phi|}^{\pi-|\theta+\phi-\pi|}\left|\mathscr{K}^{\alpha, \gamma}(\xi, \theta, \phi)\right| w^{\alpha}(\xi) d \xi \\
& \leq \max \left\{\left|\mathscr{J}_{\alpha-1 / 2}\left(\sqrt{\gamma \psi_{0}}\right)\right|:|\theta-\phi| \leq \xi \leq \pi-|\theta+\phi-\pi|\right\}
\end{aligned}
$$

by (2.7) and the argument used to prove Theorem 1, (iv). This yields (a), and (b) follows since $0 \leq \psi_{0} \leq 1$. Part (d) uses the fact that $\mathscr{I}_{\alpha-1 / 2}$ is an increasing function; parts (c) and (e) follow from slight modifications of the above.

(ii) is a simple consequence of (i)(a). 
(iii) The hypergroup assertions follow from the remarks preceding the theorem. The inequality follows since in any hypergroup, the characters must be bounded by unity.

For the radial prolate functions, when $\gamma=0, \mathscr{S}_{0}^{\alpha, 0}(z)$ has no zeros in $[0, \infty)$, and Theorem 2 yields the product formula for Jacobi functions obtained in [FK]. In this case we define

$$
d \tau_{x, y}^{\alpha, 0}(z)=\mathscr{S}_{0}^{\alpha, 0}(z)\left(\mathscr{S}_{0}^{\alpha, 0}(x) \mathscr{S}_{0}^{\alpha, 0}(y)\right)^{-1} d \sigma_{x, y}^{\alpha, 0}(z)
$$

with $d \sigma_{x, y}^{\alpha, 0}(z)=\mathscr{K}^{\alpha, 0}(z, x, y) \sinh ^{2 \alpha+1} z d z$ and $*=*^{\alpha, 0}$ analogous to the above; there is also an analogous definition of generalized translation suggested by Theorem 2 . With this definition we obtain

Theorem 4. There is a hypergroup $\left([0, \infty), *^{\alpha, 0}\right)$ with Hermitian characters $\left\{\mathscr{S}_{\lambda}^{\alpha, 0}(z) / \mathscr{S}_{0}^{\alpha, 0}(z)\right\}_{\lambda \geq 0}$, identity element $\delta_{0}$, and Haar measure $m^{\alpha, 0}$ given by $d m^{\alpha, 0}(z)=\left[\mathscr{S}_{0}^{\alpha, 0}(z)\right]^{2} \sinh ^{2 \alpha+1} z d z$. Moreover, for each $\lambda \geq 0$

$$
\left|\mathscr{S}_{\lambda}^{\alpha, 0}(z)\right| \leq \mathscr{S}_{0}^{\alpha, 0}(z) \quad(0 \leq z<\infty) .
$$

The authors are grateful to Hubert Kalf who pointed out that if $\gamma<0$ an argument based on the Sturm Comparison Theorem shows that $\mathscr{S}_{0}^{\alpha, \gamma}$ must have zeros in $(0, \infty)$, and so these methods will not yield bounds on the kernel that will allow the construction of a convolution.

\section{REFERENCES}

[AS] M. Abramowitz and I. A. Stegun, Handbook of mathematical functions, National Bureau of Standards, Washington, D.C., 1964.

[A1] F. M. Arscott, Periodic differential equations, Pergamon Press, Oxford, 1964.

[A2] _ The land beyond Bessel: A survey of higher special functions, Proc. Conf. in Ordinary and Partial Differential Equations (Dundee, 1980), (W. N. Everitt and B. D. Sleeman, eds.), Lecture Notes in Math., vol. 846, Springer-Verlag, Berlin, 1981, pp. 26-45.

[CS1] W. C. Connett and A. L. Schwartz, The theory of ultraspherical multiplies, Mem. Amer. Math. Soc. No. 183, 1977, pp. 1-92.

[CS2] _ A Hardy-Littlewood maximal inequality for Jacobi type hypergroups, Proc. Amer. Math. Soc. 107 (1989), 137-143.

[CS3] _ Analysis of a class of probability preserving measure algebras on compact intervals, Trans. Amer. Math. Soc. 317 (1990), 371-394.

[CMS] W. C. Connett, C. Markett, and A. L. Schwartz, Convolution and hypergroup structures associated with a class of Sturm-Liouville systems, Trans. Amer. Math. Soc. 332 (1992), 365-390.

[D] J. S. Dehesa, New properties of the spheroidal wave equation, Lett. Nuovo Cimento 35 (1982), 25-28.

[E] A. Erdélyi et. al, Higher transcendental functions, Vols. I and II, McGraw-Hill, New York, 1955.

[F] C. Flammer, Spheroidal wave functions, Stanford Univ. Press, Stanford, 1957.

[FK] M. Flensted-Jensen and T. H. Koornwinder, The convolution structure for Jacobi function expansions, Ark. Mat. 11 (1973), 245-262.

[G] P. R. Garabedian, Partial differential equations, Wiley, New York, 1964.

[H] P. Henrici, A survey of I. N. Vekua's theory of elliptic differential equations with analytic coefficients, Z. Angew. Math. Phys. 8 (1957), 169-203.

[I] E. L. Ince, Ordinary differential equations, Dover, 1956 (1st ed., 1926). 
[IM] M. E. H. Ismail and M. E. Muldoon, On the variation with respect to a parameter of zeros of Bessel and q-Bessel functions, J. Math. Anal. Appl. 135 (1988), 187-207.

[K] T. H. Koornwinder, Jacobi functions and analysis on noncompact semisimple Lie groups, Special Functions: Group Theoretical Aspects and Applications, (R. A. Askey, T. H. Koornwinder, and W. Schempp, eds.), Reidel, Dordrecht, 1984, pp. 1-85.

[L] E. Lanckau, Die Riemannfunktionen selbstadjungierter Gleichungen, Wiss. Z. Techn. Hochsch. Karl-Marx-Stadt 21 (1979), 535-540.

[Le] B. M. Levitan, Generalized translation operators, Israel Program for Scientific Translations, Jerusalem, 1964.

[M1] C. Markett, $A$ new proof of Watson's product formula for Laguerre polynomials via a Cauchy problem associated with a singular differential operator, SIAM J. Math. Anal. 17 (1986), 1010-1031.

[M2] _ Norm estimates for generalized translation operators associated with a singular differential operator, Indag. Math. 46 (1984), 299-313.

[M3] _ Product formulas for Bessel, Whittaker, and Jacobi functions via the solution of an associated Cauchy problem, Functional Analysis and Approximation, Proc. Conf. Oberwolfach 1983, (P. L. Butzer, R. L. Stens, and B. Sz.-Nagy, eds.), ISNM 65, Birkhäuser, Basel, 1984, pp. 449-462.

[M] N. W. Mclachlain, Theory and application of Mathieu functions, Clarendon Press, Oxford, 1947.

[Mei] J. Meixner, Integralbeziehungen zwischen Mathieuschen Funktionen, Math. Nachr. 5 (1951), 371-378.

[MS] J. Meixner and F. W. Schäfke, Mathieusche Funktionen und Sphäroid Funktionen, SpringerVerlag, Berlin, 1955.

[MSW] J. Meixner, F. W. Schäfke, and G. Wolf, Mathieu functions and spheroidal functions and their mathematical foundations, Springer-Verlag, Berlin, 1980.

[Mi] W. Miller, Jr., Symmetry and separation of variables, Encyclopaedia of Mathematics and its Applications, Vol. 4, Addison-Wesley, Reading, Mass., 1977.

[MSp] P. Moon and D. E. Spencer, Field theory handbook, Springer-Verlag, Berlin, 1961.

[P] M. Perlstadt, Polynomial analogs of prolate spheroidal wave functions and uncertainty, SIAM J. Math. Anal. 17 (1986), 240-248.

[Sc] A. L. Schwartz, The structure of the algebra of Hankel transforms and the algebra of HankelStieltjes transforms, Canad. J. Math. 23 (1971), 236-246.

[S] D. Slepian, Some comments on Fourier analysis, uncertainty, and modeling, SIAM Rev. 25 (1983), 379-393.

[SM] J. A. Stratton, P. M. Morse, L. J. Chu, J. D. C. Little, and F. J. Corbàtó, Spheroidal wave functions, Wiley, New York, 1956.

[V1] H. Volkmer, Integralrelationen mit variablen Grenzen für spezielle Funktionen der mathematischen Physik, J. Reine Angew. Math. 319 (1980), 118-132.

[V2] _ Integralgleichungen für periodische Lösungen Hillscher Differentialgleichungen, Analysis 3 (1983), 189-203.

[W] G. Watson, A treatise on the theory of Bessel functions, Cambridge Univ. Press, Cambridge, 1966.

(W. C. Connett and A. L. Schwartz) Department of Mathematics and Computer Science,

University OF Missouri-St. Louis, St. Louis, MisSouri, 63121

E-mail address: connett@umslvma.bitnet

E-mail address: schwartz@arch.umsl.edu

(Clemens Markett) Lehrstuhl A für Mathematik, Rheinisch-Westfälische Technische Hochschule Aachen, Templergraben 55, D-5100 Aachen, Germany 